ISSN: 2639-0531

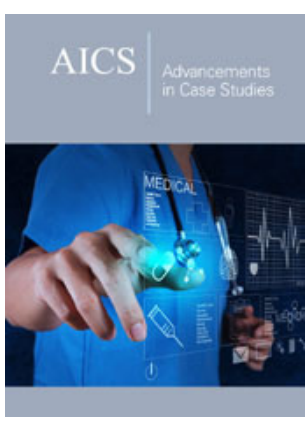

*Corresponding author: Keiko Ikemoto, Department of Psychiatry, Iwaki City Medical Center, Japan

Submission: 㽬 August 23, 2021

Published: 梅 September 15, 2021

Volume 3 - Issue 2

How to cite this article: Keiko Ikemoto. Multi-Particulate High Intensity of Brain MRI in 30's Male Heavy Smoker Suicidal Attempt Case following Mild COVID-19 Pneumonia. Adv Case Stud. 3(2). AICS.000558. 2021.

DOI: 10.31031/AICS.2021.03.000558

Copyright@ Forshing Lui, This article is distributed under the terms of the Creative Commons Attribution 4.0 International License, which permits unrestricted use and redistribution provided that the original author and source are credited.

\section{Multi-Particulate High Intensity of Brain MRI in 30's Male Heavy Smoker Suicidal Attempt Case following Mild COVID-19 \\ Pneumonia}

\author{
Keiko Ikemoto* \\ Department of Psychiatry, Iwaki City Medical Center, Japan
}

\begin{abstract}
The case report showed multi-particulate high intensity of T2-weighted image of brain MRI in 30's male heavy smoker suicidal attempt case, taken on psychiatric examination, 5 months later of his mild COVID-19 pneumonia. The MRI finding, indicating multiple micro-thrombosis is thought to be a cause of his depressed state and anosognosia.
\end{abstract}

Keywords: COVID-19; Pneumonia; Tabacco use disorder; Major depression; Suicidal attempt; Microthrombosis; MRI; Nicergoline

\section{Introduction}

Increasing number of COVID-19 patients suffer from long-lasting sequelae, not only of physical symptoms, but also of mental dysfunctions such as depression and / or cognitive impairment [1], which delay recovery processes for social re-adaptation. History of psychoses, and brain organic factors as well as newly produced psychosocial incidents, caused by COVID-19 infection, affect mental state of COVID -19 patients. Coagulation enhancementinduced thrombosis in the central nervous system (CNS) [2] is a possible organic factor of sequelae mental dysfunction including cognitive impairment. Neuropathological studies have shown that patients with fetal COVID-19 had multifocal calcifying cortical infarction, or brain stem encephalitis [3]. Whereas, it has not yet been well-known whether CNS of mild COVID-19 patients shows any characteristic morphological findings. Here the author shows brain MRI imaging with multi-particulate high intensity in a 30's male suicidal attempt case with major depressive disorder, which had a clinical history of mild COVID-19 pneumonia.

\section{Case: 30 's male}

\section{Chief complaint: suicidal attempt, left forearm cut}

Clinical history: The patient who moved alone from Tokyo area to Fukushima one year ago, after his divorce caused by large amount of debt with a slingshot, to work as a director of decontamination workers of nuclear accident in Fukushima. Five months before the present hospitalization, he received 10-day inpatient treatment of mild COVID-19 pneumonia, however, without any intraveous and oral medicine. After discharge, he took one month home recuperation, and reworked. Persistent dysgeusia and smell disorder continued. One day, a colleague who failed to get in touch with the patient's cell phone, visited his room and found him in a bathroom with his left wrist cut with a kitchen knife. 
Clinical course: At the hospital, his left forearm cut injury with dissection of medial and radial nerves, radial artery and several tendons, due to self-mutilation and suicide attempt, was satured in microsurgery. He smoked 40 cigarettes a day and had alcohol opportunity drinking. The 3rd day of his hospitalization, a liaisonconsultation-psychiatrist examined him, and found him in his depressed state with suicidal idea, lacking insight of his depressed mood and social dysfunction, implying a sort of anosognosia. No abnormal findings in thyroid pituitary adrenal function test. CES-D (The Center of Epidemiologic Studies Depression Scale) score 29, that is mood disorder.
Psychiatric pharmacotherapy was initiated by using antidepressant, $15 \mathrm{mg}$ of mirtazapine. Brain imaging of CT showed slight asymmetry in frontal white matter low density $(\mathrm{L}>\mathrm{R})$, and T2-weighted image of brain MRI showed multi-particulate high intensity pattern ( $\mathrm{L}>\mathrm{R}$ ), indicating micro-thrombosis (Figure 1). Then, cerebral circulation improving drug, $15 \mathrm{mg}$ of Nicergoline was additionally prescribed. His depressed state improved, and discharged to return to home in Tokyo area, within 11 days. A brain organic factor shown in multi-particulate high intensity of parietal region in MRI, was thought to be a cause of his depressive state and anosognosia. The psychiatric diagnosis was major depressive disorder, gambling disorder and tabacco use disorder.

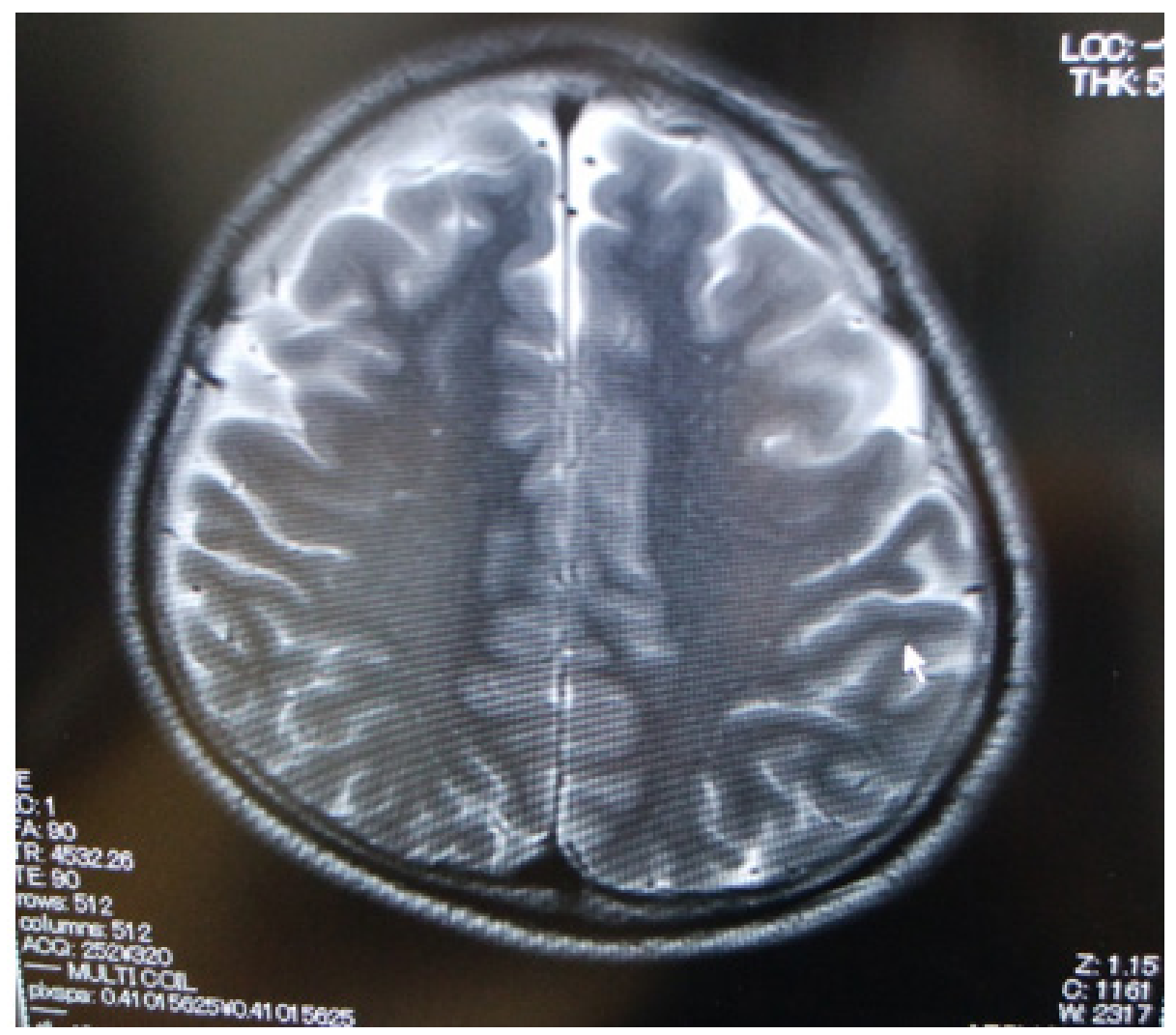

Figure 1: Microthrombosis-like multi-particulate high intensity ( $\mathrm{L}>\mathrm{R})$ seen in T2-weighted image of brain MRI of a 30's male heavy smoker suicide attempt case, 5 months later of mild COVID-19 pneumonia without requiring any medication.

\section{Discussion}

The unique brain MRI pattern of multi-particulate high intensity, despite his young's age, following his suicidal attempt, is thought to be multi-thrombosis, the result of coagulation enhancement due to COVID-19 infection [2,4], though pneumonia symptoms were mild. He had not yet been vaccinated and did not take any medication for COVID-19 treatment, including heparin, which may prevent vascular thrombosis [4]. His daily habit of a heavy smoker and gambling disorder might lead his earlier infection by COVID-19 [5]. Nicergoline, a cerebral circulation improving drug, was a possible agent effective for depressed state and cognitive impairment, following COVID-19.

\section{Conclusion}

In cases of post-COVID-19 depression and cognitive impairment, brain image examination is important to detect vascular elements.

\section{References}

1. Huang C, Huang L, Wang Y, Li X, Ren L, et al. (2021) 6-month consequences of COVID-19 in patients discharged from hospital: a cohort study. Lancet 397(10270): 220-232. 
2. Boyd S, Martin-Loeches I (2021) The incidence of venous thromboembolism in critically ill patients with COVID-19 compared with critically ill non-COVID patients. Ir J Med Sci 7: 1-4.

3. Jensen MP, Le Quesne J, Officer-Jones L, Teodosio A, Thaventhiran J et al (2021) Neuropathological findings in two patients with fetal COVID-19. Neuropathol Appl Neurobiol 47(1): 17-25
4. Asakura H, Ogawa H (2021) COVID-19-associated coagulopathy and disseminated intravascular coagulation. Int J Hematol 113(1):45-57.

5. Van Zyl-Smit RN, Richards G, Leone FT (2020) Tobacco smoking and COVID-19 infection. Lancet Respir Med 8(7): 664-665.

For possible submissions Click below:

Submit Article 